\title{
Edukasyon sa Bagong Kadawyan: Hamon sa pagsagot ng modyul ng mga mag-aral na nagpapakadalubhasa sa Filipino
}

\author{
Urgil, Nelson $\bowtie$ \\ Leyte Normal University, Philippines (nelsonurgilc@gmail.com)
}

Aninao, John Lester

Leyte Normal University, Philippines (johnlesteraninao@gmail.com)

Santo, Hannah

Leyte Normal University, Philippines (hannahsanto18@gmail.com)

Silvano, Leslie

Leyte Normal University, Philippines (lessilvano20@gmail.com)

Uñalan, Leiah Anne

Leyte Normal University, Philippines (haiel.unalan2580@gmail.com)

Received: 5 November 2021

Available Online: 2 January 2022

Revised: 29 November 2021 DOI: 10.5861 /ijrse. 2022.210

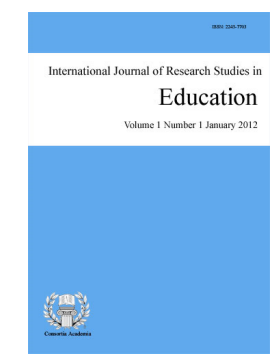

ISSN: $2243-7703$ Online ISSN: 2243-7711

OPEN ACCESS

\section{Abstract}

Online learning is the new normal in education. With this, modules (printed and electronic) are used in the delivery of lessons. This research was conducted to identify the problems encountered by the students in answering these modules as one of the primary learning materials used in an online education. Also, this seeks to identify some interventions to help students in their current situation. This is a quantitative research using a cross-sectional design that targets to elicit responses that will satisfy the research objectives. A structured survey questionnaire was prepared and distributed to 80 respondents to represent the population which were selected randomly. The number of respondents was determined using the table of population and sample size by Krejcie and Morgan (1970). Based on the gathered data, the researchers found that the students experienced difficulty in answering the modules due to lack of digital tools/gadgets and other resources that can be used to further understand the lesson. Internet connections and the learning environment are also factors that makes online learning difficult. Also, answering these modules become difficult to the students because they don't have someone to ask and help them to understand the concepts. With these, the researchers recommended that teachers become more flexible and understanding in terms of establishing academic rules and requirements in his/her respective subject. For tasks and requirements, it is necessary to give sufficient time and/or day to the students to complete these modules and for teachers to aid students' learning by employing both asynchronous and synchronous teaching.

Keywords: new normal, education, difficulties, answering, module 


\section{Edukasyon sa Bagong Kadawyan: Hamon sa pagsagot ng modyul ng mga mag-aral na nagpapakadalubhasa sa Filipino}

\section{Introduksiyon}

Sa kasalukuyang sistema ng edukasyon, maraming suliranin ang kinahaharap ng kaguruan, magulang at mag-aaral sa ModularLearning (printed at electronic) na pangunahing ginagamit ng mga guro at mag-aaral sa elementarya, sekondarya, at kolehiyo. Malaki ang pinagkaiba ng pagtuturo sa face-to-face sapagkat dito ay epektibong nagagamit ang iba't ibang estratehiya sa pagtuturo-pagkatuto at dito rin ay mayroong agarang gabay mula sa guro. Samakatuwid, sa pamamaraang modular learning ay nakasalalay ang pagkatuto ng mga mag-aaral sa kanilang sarili at paggabay mula sa kanilang mga magulang, kapatid, kaklase at/o kaibigan para sa pag-unawa ng mga konsepto. Sa pag-aaral na isinagawa ni Pe Dangle et al. (2020), binigyang-tuon ang mga suliranin na nararanasan ng mga estudyante, magulang, at guro pagdating sa oras, kagamitan, at internet connection. Lumabas sa pag-aaral na ang mga estudyante ay nahihirapan sa pamamaraang modular learning at ang mga magulang ay walang sapat na kaalaman sa mga konseptong nakapaloob sa modyul. Lumabas din sa pag-aaral nina Musingafi et al. (2015), na ang suliraning nararanasan ng mga estudyante sa pamamaraang open and distance learning ay aang kakulangan sa oras, nahihirapan sa internet connectionat paggamit ng ICT, ang pagbibigay ng pidbak sa pagitan ng estudyante at ng guro ay hindi epektibong nagaganap, at higit sa lahat kulang sa kagamitan ang mga estudyante tulad ng aklat at gadgets.

Lumabas sa pag-aaral nina Kedraka at Kaltsidisi (2020), na ang mga nasa malayong lugar ay hindi magagawang makasabay sa mga gawain lalo na kung ang mga ito ay walang gamit at internet connection na kapag nagpatuloy ang ganitong pamamaraan ay mas lumalakas ang digital gap na kung saan hindi lahat ng mag-aaral ay may ginagamit na gadgets at may mabilis na internet connections.

Sa pag-aaral nina Olamo et al. (2019), karamihan sa mga instruktor ay naglalaan ng oras at ang implementasyong ito ay epektibong naipapatupad sa pamamagitan ng mga inirekomendang paraan ng pagtuturo, pagtatasa ng kaalaman ng mga estudyante gamit ang wastong pamamaraan o estratehiya, at sa pamamagitan na rin ng mga gabay at tulong ng mga instruktor sa kanilang mga estudyante. Ganoon pa man, mayroon pa ring mga instruktor na hindi binigyang-pokus ang implementasyong ito, walang pakialam, at hindi iniisip ang pagkatuto ng mga estudyante.

Ganoon din sa pag-aaral nina Karal et al. (2011), lumabas sa pag-aaral na ang mga suliraning nangyayari sa distance education partikular sa online na pagsusulit ay pagdating sa antas ng kaalaman sa paggamit ng kompyuter, nahihirapan ang estudyante lalo na kung ang pamamaraang ito ay bago sa kanila, nagkakaroon din ng problema sa paraan ng pagkakasulat at pagkakabuo ng mga tanong, at ang technical problems na kadalasang ito ay nangyayari at ang ganitong suliranin ay hindi naiiwasan. Sa pag-aaral na ito, ang pinakamahalagang resulta na kanilang natuklasan ay kailangang magsagawa ng programa at oryentasyon patungkol sa online na pagsusulit at paggamit ng kompyuter sapagka't ito'y napakahalagang salik upang matugunan ang mga nasabing suliranin.

Ayon din kay Vonderwell (2003), na ang online learning napakaraming mga suliranin at pagsubok na kailangan harapin kabilang na rito ang kakulangan ng interaksyon mula sa mga mag-aaral at mga guro kaya ang ganitong pamamaraan ang pagkatuto ay nakasalalay sa pagsisikap ng bawat mag-aaral. Dagdag pa ni Nardo (2017), na ang ganitong uri ng sistema sa pagtuturo ang dahilan kung bakit nahahasa ng mga mag-aaral ang responsableng pagkatuto sa mga gawaing nakapaloob sa kanilang modyul.

Nakikita ng mga mananaliksik na ang pag-aaral na ito ay mahalaga sa kalipunan ng kalaaman at maaring gamitin bilang mainam na batayan sa pagbuo ng mga metodo o estratehiya, at maging sa polisiyang 
Edukasyon sa Bagong Kadawyan: Hamon sa pagsagot ng mga mag-aral na nagpapakadalubhasa sa Filipino pangkurikulum partikular na sa patuloy na pagpapatupad ng online learning. Sa kabilang banda, mainam din ang pag-aaral na ito bilang gabay sa mga guro at mag-aaral nang sa ganoon ay mas mapapadali at mapapagaan ang pagtuturo at pagkatuto sa paraang modular learning sa bagong kadawyan.

\subsection{Kaligirang teoitikal}

Ang online learning ay nangyayari sa pamamaraang synchronous at asynchronous o maaari ring sa parehong pamamaraan. Ang synchronous na pamamaraan ay ang mga guro at mag-aaral gamit ang live chatting or live video conference. Dagdag pa, ang guro ay agarang nakapagbibigay ng tugon mula sa mga katanungan ng mga mag-aaral. Samantala, ang asynchronous na pamamaraan naman ay ang paggamit ng recorded video lessons na mapapanuod ng mga mag-aaral sa oras na gustuhin nila. Kaugnay nito, sa online learning sa antas ng kolehiyo ang pinaka-apektado sa pamamaraang ito ang mga mag-aaral na nasa unang taon. Ito ay pinatunayan sa pag-aaral nina O’Brien at Verama (2018), na kung saan nalaman nila na ang mga mag-aaral na nasa unang taon ang nagtataglay ng maraming salik na dapat isaalang-alang gaya ng antas ng kaalaman, kasarian, edad at antas ng pagkatuto na makakaapekto sa kalidad ng kaalaman sa paraang online learning. Sinang-ayunan ito nina Handel et al. (2020), na lumabas sa kanilang pagsusuri na bukod sa tensyong nararanasan ng mga mag-aaral ay kasama pa rito ang kakulangan sa kagamitan at kakulangan sa kaalaman sa paraang online learning.

Sa pag-aaral na ito ginamit ang Moore's Transactional Distance Theory. Binuo ni Moore ang teoryang ito upang suriin ang dalawang baryabol: "pamamaraan ng estudyante" at "komunikasyon ng estudyante at ng guro" (Hanson et al. 1997). Ayon kay Moore ang paniniwalang ito ay ibinatay sa distance learning ayon sa sosyal na proseso ng pagtuturo hindi ibinatay sa tradisyunal na konteksto. Ginamit din ang Media Richness Theory na binuo nila Daft at Lengel noong 1986 kung saan ang teoryang ito ay nagpapaliwanag na malaki ang naitutulong ng mobile technologies sa larangan ng edukasyon kung saan ito ay nagsisilbing kaparaanan ng komunikasyon at interaksiyon ng bawat indibidwal. Samakatuwid, nahaharap naman sa malaking suliranin ang isang indibidwal kung wala silang kagamitan/gadgets na magagamit sa kanilang pag-aaral (Sarrab, 2015).

Binigyang-diin din ng pananaw na ito na ang mobile richness ay nakakatulong upang hindi mahirapan sa pag-unawa ang mga estudyante sa mga konsepto at sa pamamagitan rin nito ay epektibong natututo ang estudyante (Almutairy et al. 2015). Samakatuwid, kung kulang o walang teknolohiyang ginagamit ang isang estudyante at ang guro ay siguradong kulang at hindi epektibo ang pagkatuto-pagtuturo.

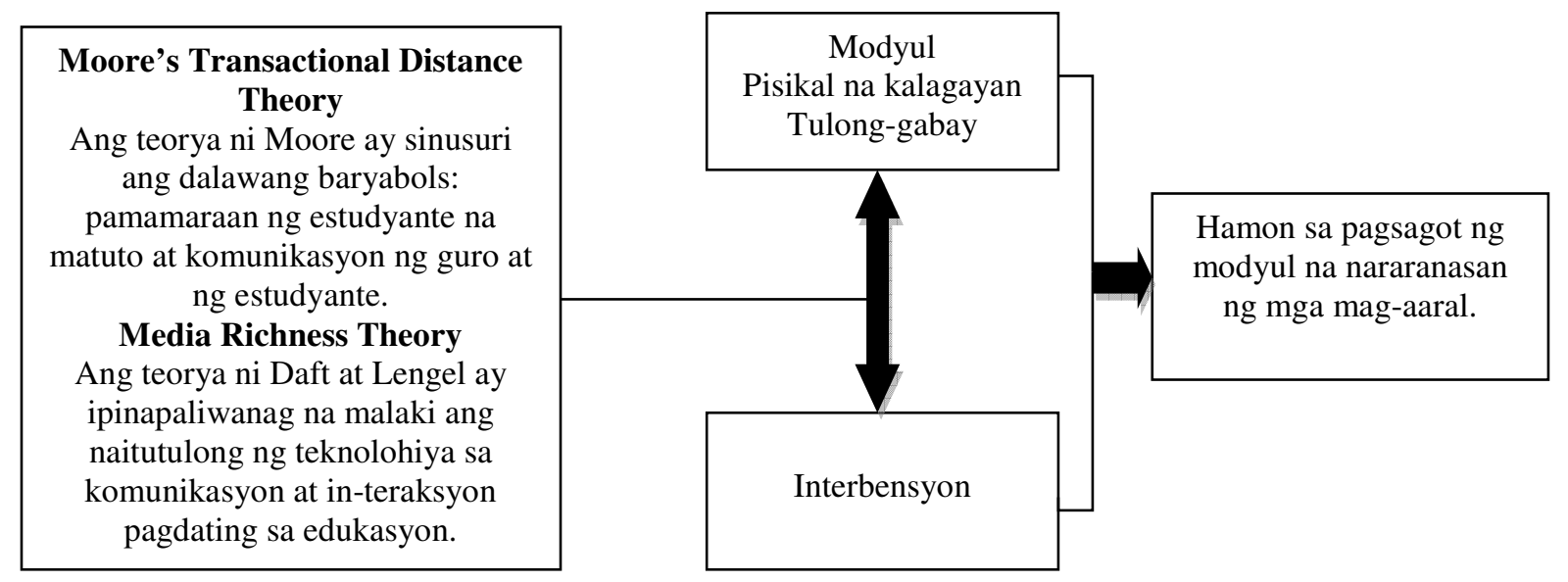

Figyur 1. Balangkas ng Teoritikal ng Pag-aaral

Sa Figyur 1 makikita ang balangkas ng pag-aaral, makikita sa unang kahon ang mga teoryang ginamit, ang Moore's Transactional Distance Theory at ang Media Richness Theory. Ang dalawang kahon na makikita sa gitna ay ang mga baryabol: modyul, pisikal na kalagayan, tulong-gabay, at interbensyon. Sa huling bahagi ay 
Urgil, N., Aninao, J. L., Santo, H., Silvano, L., \& Uñalan, L. A.

malalaman ang resulta ng pag-aaral patungkol sa hamon sa pasagot ng modyul na nararanasan ng mga mag-aaral na nagpapakadalubhasa sa Filipino ng Leyte Normal University.

\subsection{Paglalahad ng suliranin}

Ang pananaliksik na ito ay naglalayong malaman ang hamon sa pasagot ng modyul ng mga mag-aaral na nagpapakadalubhasa sa Filipino ng Leyte Normal University. Tinitiyak ng pananaliksik na bigyang-kasagutan ang sumusunod na Tanong: (1) Ano-ano ang suliraning kinakaharap ng mga mag-aaral sa pagsagot ng modyul batay sa; (a) modyul (b) pisikal na kalagayan (c) tulong-gabay? at (2) Ano-ano ang interbensyon sa mga suliraning kinakaharap ng mga mag-aaral sa pagsagot ng modyul?

\section{Metodolohiya}

Ang pag-aaral na ito ay kwantitatibo na gumamit ng disenyong palarawang sarbey o deskriptibong pananaliksik na sumasaklaw sa paglalarawan at pagsusuri ng mga datos at paglalaan ng maliwanag na pag-unawa tungkol sa hamon sa pagsagot ng modyul na kinakaharap ng mga mag-aaral. Ang sakop ng pag-aaral na ito ay ang mga mag-aaral na nagpapakadalubhasa sa Filipino, unang taon sa kolehiyo ng Leyte Normal University, Tacloban City. Ang respondente ng pag-aaral ay mula sa unang taon na nagpapakadalubhasa sa Filipino sa Leyte Normal University, Tacloban City na mayroong kabuuang populasyon na 100. Ginamit ng mananaliksik ang talahanayan nina Krejcie at Morgan (1970) sa pagkuha ng sample size na kung saan mayroong kabuuang walumpu (80) na tiyak na kinatawan ng populasyon. Samantala, sa pagtukoy ng mga respondente ay ginamit ng mananaliksik ang non-equal random sampling kung saan ang lahat ng mga nagpapakadalubhasa sa Filipino sa unang taon ay may pagkakataon na maging respondente sa pag-aaral at upang mapanatili ang pagiging obhektibo gumamit ang mananaliksik ng fish-bowl method sa pagkuha ng mga pangalan sa nasabing walumpung (80) mag-aaral na sasagot sa katanungan na nakapaloob sa sarbey kwestyuner. Ang instrumentong ginamit sa pag-aaral ay ang talatanungan o sarbey kwestyuner. Gumamit ang mananaliksik ng LikertScale atsa kompyutasyon ng bahagdan ay ginamit ang simple percentage at weighted mean na pamamaraan.

\section{Talahanayan I}

Likert Scale

\begin{tabular}{cccc}
\hline Likert Scale & Interval & Difference & Numerical Value \\
\hline 5 & $4.20-5.00$ & 0.80 & Lubos na sumasang-ayon \\
4 & $3.40-4.19$ & 0.79 & Sumasang-ayon \\
3 & $2.60-3.39$ & 0.79 & Walang katiyakan \\
2 & $1.80-2.59$ & 0.79 & Hindi sumasang-ayon \\
1 & $1.00-1.79$ & 0.79 & Lubos na hindi sumasang-ayon \\
\hline
\end{tabular}

\section{Mga Resulta at Diskusyon}

Ang bahaging ito ay tumatalakay sa nakalap na datos tungkol sa mga suliranin sa pagsagot ng modyul na nararanasan ng mga mag-aaral na nagpapakadalubhasa sa Filipino partikular sa modyul, pisikal na kalagayan, at tulong-gabay.

\subsection{Ang mga suliraning kinakaharap ng mga mag-aaral sa pagsagot ng modyul batay sa:}

Ang talahanayan ay nagpapakita ng resulta ng mga nakalap na datos. Batay sa ipinapakita ng talahanayan, ang unang pahayag ay mayroong weighted mean na 4.4125 na may diskripsyong lubos na sumasang-ayon. Sa ikalawang pahayag naman ay mayroong weighted mean na 4.4375 na may diskripsyong lubos na sumasang-ayon. Samantala, ang pangatlong pahayag ay mayroong weighted mean na 4.1 kung saan ang katumbas na diskripsyon ay sumasang-ayon. Ang pang-apat na pahayag naman ay may weighted mean na 4.1625 na may diskripsyong sumasang-ayon, at ang ikalimang pahayag ay mayroong weighted mean na 4.0375 na may diskripsyong sumasang-ayon. Ibig sabihin ang mga mag-aaral na nasa unang taon na nagpapakadalubhasa sa Filipino ay hindi

90 Consortia Academia Publishing (A partner of Network of Professional Researchers and Educators) 
Edukasyon sa Bagong Kadawyan: Hamon sa pagsagot ng mga mag-aral na nagpapakadalubhasa sa Filipino nakararanas ng mga suliraning nakasaad hinggil sa pisikal na anyo (disenyo) ng modyul.

\section{Talahanayan 2}

Suliranin sa pagsagot ng modyul: Disenyo

\begin{tabular}{lcc}
\hline \multicolumn{1}{c}{ Pahayag } & Weighted Mean & Deskripsyon \\
\hline Malinaw at maayos ang mga larawang ginamit sa modyul. & 4.4125 & Lubos na sumasang-ayon \\
Ang mga kulay na ginamit ay hindi masakit sa mata. & 4.4375 & Lubos na sumasang-ayon \\
Malinaw at malaki ang pagkakasulat sa mga salita. & 4.1 & Sumasang-ayon \\
Hindi magkakadikit-dikit ang mga salita/pangungusap. & 4.1625 & Sumasang-ayon \\
Iisa ang istilo/style na ginamit sa mga salita. & 4.0375 & Sumasang-ayon \\
\hline
\end{tabular}

\section{Talahanayan 3}

Suliranin sa Pagsagotng Modyul: Paksa at Nilalaman

\begin{tabular}{|c|c|c|}
\hline $\begin{array}{l}\text { Pahayag } \\
\end{array}$ & Weighted Mean & Diskripsyon \\
\hline Ang bawat bahagi sa modyul ay buo at kumpleto. & 4.325 & Lubos na sumasang-ayon \\
\hline Madaling maunawaan ang lahat ng konseptong tinalakay. & 3.7125 & Sumasang-ayon \\
\hline $\begin{array}{l}\text { Sapat at kaiga-igaya ang pagtalakay sa bawat paksa at mga } \\
\text { gawain. }\end{array}$ & 4 & Sumasang-ayon \\
\hline $\begin{array}{l}\text { Ang mga impormasyon ay tumpak at may kaugnayan sa } \\
\text { paksang tinalakay. }\end{array}$ & 4.525 & Lubos na sumasang-ayon \\
\hline $\begin{array}{l}\text { Ang mga salitang ginamit ay hindi malalim at madaling } \\
\text { maunawaan. }\end{array}$ & 3.9625 & Sumasang-ayon \\
\hline
\end{tabular}

Batay sa ipinapakita ng talahanayan, ang unang pahayag ay mayroong weighted mean na 4.325 na may diskripsyong lubos na sumasang-ayon. Sa ikalawang pahayag naman ay mayroong weighted mean na 3.7125 na may diskripsyong sumasang-ayon. Samantala, ang pangatlong pahayag ay mayroong weighted mean na 4 kung saan ang katumbas na diskripsyon ay sumasang-ayon. Ang pang-apat na pahayag naman ay may weighted mean na 4.525 na may diskripsyong lubos na sumasang-ayon, at ang ikalimang pahayag ay mayroong weighted mean na 3. 9625 na may diskripsyong sumasang-ayon. Ibig sabihin ang mga mag-aaral na nasa unang taon na nagpapakadalubhasa sa Filipino ay hindi nararanasan ang mga suliraning nakasaad patungkol sa paksa at nilalaman

\section{Talahanayan 4}

Suliranin sa Pagsagot ng Modyul: Gawain at Kahingian

\begin{tabular}{lcc}
\hline \multicolumn{1}{c}{ Pahayag } & Weighted Mean & Diskripsyon \\
\hline $\begin{array}{l}\text { Malinaw ang mga panuto sa bawat gawain o pagsasanay. } \\
\text { Madaling sagutan ang lahat ng mga gawain sa bawat paksa } \\
\text { at nagsisimula sa madali hanggang sa mahirap. }\end{array}$ & 4.1125 & $\begin{array}{l}\text { Sumang-ayon } \\
\text { Sumasang-ayon }\end{array}$ \\
$\begin{array}{l}\text { Ang mga gawain ay may kaugnayan/angkop sa mga } \\
\text { layunin ng pagtuturo. }\end{array}$ & 3.9625 & Lubos na sumasang-ayon \\
$\begin{array}{l}\text { Ang mga gawain ay may krayteryang sinusunod. } \\
\text { Ang lahat ng mga gawain ay kayang gawin ng mag-isa. }\end{array}$ & 4.3625 & Lubos na sumasang-ayon \\
Ang mga gawain ay naisaalang-alang ang kakayahan at & 4.5 & $\begin{array}{l}\text { Sumasang-ayon } \\
\text { Sumasang-ayon }\end{array}$ \\
Angayan ng estudyante. & 3.4125 & Sumasang-ayon \\
\hline
\end{tabular}

Makikita sa talahanayan na ang unang pahayag ay mayroong weighted mean na 4.1125 na may diskripsyong sumasang-ayon. Sa ikalawang pahayag naman ay mayroong weighted mean na 3.9625 na may diskripsyong sumasang-ayon. Samantala, ang pangatlong pahayag ay mayroong weighted mean na 4.3625 na may diskripsyong lubos na sumasang-ayon. Ang pang-apat na pahayag naman ay may weighted mean na 4.5 na may diskripsyong lubos na sumasang-ayon, at ang ikalimang pahayag ay mayroong weighted mean na 3.4125 na may diskripsyong sumasang-ayon. Sa ika-anim na pahayag naman ay may weighted mean na 3.9625 na may diskripsyong sumasang-ayon, at ang huling pahayag naman ay may weighted mean na 3.45 na may diskripsyong sumasang-ayon. Ibig sabihin ang mga mag-aaral na nasa unang taon na nagpapakadalubhasa sa Filipino ay hindi nararanasan ang mga suliranin patungkol sa gawain at kahingian. 
Urgil, N., Aninao, J. L., Santo, H., Silvano, L., \& Uñalan, L. A.

\section{Talahanayan 5}

Suliranin sa Pagsagotng Modyul: Oras

\begin{tabular}{lcc}
\hline \multicolumn{1}{c}{ Pahayag } & Weighted Mean & Diskripsyon \\
\hline $\begin{array}{l}\text { Nagaganap ang pagtuturo-pagkatuto sa bawat oras/iskedyul ng } \\
\text { klase. }\end{array}$ & 3.725 & Sumasang-ayon \\
$\begin{array}{l}\text { Nakukuha/natatanggap ang modyul sa itinakdang araw/oras. } \\
\text { Sapat ang oras na inilaan para masagutan ang lahat ng gawain at }\end{array}$ & 3.8625 & $\begin{array}{l}\text { Sumasang-ayon } \\
\text { pagsasanay na nakapaloob sa modyul. }\end{array}$ \\
$\begin{array}{l}\text { Nakakapaglaan ng oras upang mapag-aralan ang mga konsepto. } \\
\text { Naisusumite ang sinagutang modyul sa itinakdang oras. }\end{array}$ & 3.9125 & Sumanon \\
\hline
\end{tabular}

Ang talahanayan ay resulta ng mga nakalap na datos. Sa unang pahayag ay mayroong weighted mean na 3.725 na may diskripsyong sumasang-ayon. Ang weighted mean naman ng ikalawang pahayag ay 3.8625 na may diskripsyong sumasang-ayon. Samantala, ang pangatlong pahayag ay mayroong weighted mean na 3.9125 kung saan ang katumbas na diskripsyon ay sumasang-ayon. Ang pang-apat na pahayag naman ay may weighted mean na 4.05 na may diskripsyong sumasang-ayon, at ang ikalimang pahayag ay mayroong weighted mean na 4.0125 na may diskripsyong sumasang-ayon. Ibig sabihin ang mga mag-aaral nanasa unang taon na nagpapakadalubhasa sa Filipino ay hindi nararanasan ang mga suliranin nakasaad patungkol sa oras.

\section{Talahanayan 6}

Suliranin sa pagsagot ng modyul: Kagamitan/Gadgets

\begin{tabular}{lcc}
\hline \multicolumn{1}{c}{ Pahayag } & Weighted Mean & Diskripsyon \\
\hline $\begin{array}{l}\text { Humihiram ng cellphone/laptop sa kapit-bahay/kaibigan. } \\
\begin{array}{l}\text { Cellphone ang ginagamit sa pagsagot/paggawa sa mga } \\
\text { gawain. }\end{array}\end{array}$ & 2.9625 & Walang katiyakan \\
$\begin{array}{l}\text { Laptop at cellphone ang ginagamit sa pagsagot sa mga } \\
\text { gawain }\end{array}$ & 3.3375 & Subos na sumasang-ayon \\
$\begin{array}{l}\text { May aklat na napagkukunan ng mga impormasyon } \\
\text { May personal-wifi akong ginagamit sa bahay. }\end{array}$ & 3.5875 & Hindi sumasang-ayon \\
\hline
\end{tabular}

Makikita sa talahanayan na ang unang pahayag ay mayroong weighted mean na 2.9625 na may diskripsyong walang katiyakan. Gayunpaman, ang ikalawang pahayag ay mayroong weighted mean na 4.3375 na may diskripsyong lubos na sumasang-ayon. Sa ikatlong pahayag naman ay mayroong weighted mean na 3.5875 kung saan ang katumbas na diskripsyon ay sumasang-ayon. Samakatuwid, ang ikaapat at ikalimang pahayag ay mayroong diskripsyon na hindi sumasang-ayon kung saan ang ikaapat na pahayag ay may weighted mean na 2.4375 at ang ikalimang pahayag ay mayroong weighted mean na 2.5625. Batay sa datos, ang mga mag-aaral na nasa unang taon na nagpapakadalubhasa sa Filipino ay mayroong suliranin pangkagamitan na kinakaharap.

\section{Talahanayan 7}

Suliranin sa Pagsagot ng Modyul: Internet Connection

\begin{tabular}{lcc}
\hline \multicolumn{1}{c}{ Pahayag } & Weighted Mean & Diskripsyon \\
\hline Stable/malakas ang signal sa aming lugar. & 2.5875 & Hindi sumasang-ayon \\
Walang internet connection sa aming lugar. & 2.425 & Hindi sumasang-ayon \\
Kailangang lumabas ng bahay upang makakuha ng signal. & 3.375 & Walang katiyakan \\
Nakiki-connect lamang sa aming kapitbahay. & 2.2 & Hindi sumasang-ayon \\
Peso-Vendo-Wifi ang ginagamit sa internet connection. & 2.2375 & Hindi sumasang-ayon \\
\hline
\end{tabular}

Batay sa ipinapakita ng talahanayan, ang unang pahayag ay mayroong weighted mean na 2.5875 na may diskripsyong hindi sumasang-ayon, sa ikalawang pahayag naman ay may weighted mean na 2.425 na may diskripsyong hindi sumasang-ayon, ang pangatlong pahayag naman ay may weighted mean na 3.375 na may diskripsyong walang katiyakan, ang pang-apat na pahayag naman ay may weighted mean 2.2 na may diskripsyong hindi sumasang-ayon, at ang huling pahayag naman ay may weighted mean na 2.2375 na may diskripsyong hindi sumasang-ayon. Ibig sabihin ang mga mag-aaral na nasa unang taon na nagpapakadalubhasa sa Filipino ay may suliraning nararanasan pagdating sa internet connection. 
Edukasyon sa Bagong Kadawyan: Hamon sa pagsagot ng mga mag-aral na nagpapakadalubhasa sa Filipino

\section{Talahanayan 8}

Suliranin sa Pagsagot ng modyul: Kapaligiran (Environment)

\begin{tabular}{lcc}
\hline \multicolumn{1}{c}{ Pahayag } & Weighted Mean & Diskripsyon \\
\hline Tahimik ang bahay tuwing ako ay nag-aaral. & 2.525 & Hindi sumasang-ayon \\
Mula sa kapitbahay ay walang-ingay na maririnig. & 2.3875 & Hindi sumasang-ayon \\
Ligtas, at malinis ang lugar na aking kinalalagyan. & 3.95 & Sumasang-ayon \\
Hindi mainit/tumutulo ang lugar na aking kinalalagyan. & 3.5125 & Sumasang-ayon \\
Malapit/mabilis makakuha sa mga learning materials & 2.8125 & Walang katiyakan \\
(availability of learning materials like books, and computer & & \\
shops). & & \\
\hline
\end{tabular}

Makikita sa talahanayan na ang unang pahayag ay mayroong weighted mean na 2.525 na may diskripsyong hindi sumasang-ayon. Sa ikalawang pahayag naman ay mayroong weighted mean na 2.3875 na may diskripsyong hindi sumasang-ayon. Samantala, ang pangatlong pahayag ay mayroong weighted mean na 3.95 kung saan ang katumbas na diskripsyon ay sumasang-ayon. Ang pang-apat na pahayag naman ay may weighted mean na 3.5125 na may diskripsyong sumasang-ayon, at ang ikalimang pahayag ay mayroong weighted mean na 2.8125 na may diskripsyong walang katiyakan. Ibig sabihin ang mga mag-aaral na nasa unang taon na nagpapakadalubhasa sa Filipino ay nakararanas ng suliranin pangkapaligiran/environment.

\section{Talahanayan 9}

Suliranin sa pagsagot ng modyul: Magulang

\begin{tabular}{lcc}
\hline \multicolumn{1}{c}{ Pahayag } & Weighted Mean & Diskripsyon \\
\hline Nakapagtapos ang aking magulang sa kanyang pag-aaral. & 2.65 & Walang katiyakan \\
Naglalaan ng oras upang ako'y gabayan at turuan. & 2.725 & Walang katiyakan \\
Naipapaliwanag nang maayos ang mga konseptong hindi & 2.8375 & Walang katiyakan \\
ko lubos maunawaan. & & \\
Tumutulong sa pagsagot/pagbuo sa mga proyekto/gawain. & 2.5625 & Hindi sumasang-ayon \\
Naglalaan ng mga aktibidad sa pagkatuto o mabilisang & 2.9 & Walang katiyakan \\
pagkaunawa sa mga konsepto. & & \\
\hline
\end{tabular}

Ang talahanayan ay resulta ng mga nakalap na datos. Batay sa ipinapakita ng talahanayan, ang una, pangalawa, pangatlo, at panlimang pahayag ay mayroong diskripsyong walang katiyakan. Ganoon pa man, ang unang pahayag ay mayroong weighted mean na 2.65 , ang pangalawa ay 2.725 , pangatlo ay 2.5625 , ang panlima ay 2.9. Samakatuwid, ang pang-apat na pahayag ay mayroong 2.5625 weighted mean na may diskripsyong hindi sumasang-ayon. Sa kabuuan, ang mga mag-aaral na nasa unang taon na nagpapakadalubhasa sa Filipino ay hindi nakatatanggap ng tulong-gabay galing sa kanilang mga magulang.

\section{Talahanayan 10}

Suliranin sa Pagsagot ng Modyul: Kapatid

\begin{tabular}{lcc}
\hline \multicolumn{1}{c}{ Pahayag } & Weighted Mean & Diskripsyon \\
\hline Nakapagtapos ang aking kapatid sa kanyang pag-aaral. & 2.9875 & Walang katiyakan \\
Tumutulong sa pagsagot/pagbuo sa mga proyekto/gawain. & 2.5 & Hindi sumasang-ayon \\
$\begin{array}{l}\text { Naipapaliwanag nang maayos ang mga konseptong hindi } \\
\text { ko lubos maunawaan. }\end{array}$ & 2.5875 & Hindi sumasang-ayon \\
$\begin{array}{l}\text { May oras siyang ako ay gabayan at turuan. } \\
\begin{array}{l}\text { Naglalaan ng mga aktibidad sa pagkatuto o mabilisang } \\
\text { pagkaunawa sa mga konsepto. }\end{array}\end{array}$ & 2.3 & Hindi sumasang-ayon \\
\end{tabular}

Ang talahanayan ay resulta ng mga nakalap na datos. Batay sa ipinapakita ng talahanayan, ang unang pahayag ay mayroong weighted mean na 2.9875 na may diskripsyong walang katiyakan. Ganoon pa man, mula sa talahanayan ay makikita na ang ikalawa, ikatlo, ikaapat, at ikalimang pahayag ay mayroong diskripsyong hindi sumasang-ayon. Datapwat, ang ikalawang pahayag ay mayroong weighted mean na 2.5, ang pangatlo ay 2.5875, ang pang-apat ay 2.3, at ang panglima ay 2.35. Mula sa datos ay masasabi na ang mga mag-aaral na nasa unang taon na nagpapakadalubhasa sa Filipino ay hindi sila natutulungan ng kanilang kapatid sa pagsagot ng modyul. 
Urgil, N., Aninao, J. L., Santo, H., Silvano, L., \& Uñalan, L. A.

\section{Talahanayan 11}

Suliranin sa pagsagot ng modyul: Kaibigan

\begin{tabular}{lcc}
\hline \multicolumn{1}{c}{ Pahayag } & Weighted Mean & Diskripsyon \\
\hline Nakapagtapos ang aking kaibigan sa kanyang pag-aaral. & 2.8375 & Walang katiyakan \\
Tumutulong sa pagsagot/pagbuo sa mga proyekto/gawain. & 3.0375 & Walang katiyakan \\
Naipapaliwanag ng maayos ang mga konseptong hindi ko & 3.1875 & Walang katiyakan \\
lubos maunawaan. & & \\
Naglalaan ng oras upang ako ay gabayan at turuan. & 2.9625 & Walang katiyakan \\
Naglalaan ng mga aktibidad sa pagkatuto o mabilisang & 2.825 & Walang katiyakan \\
pagkaunawa sa mga konsepto. & & \\
\hline
\end{tabular}

Batay sa ipinapakita ng talahanayan, ang unang pahayag ay mayroong weighted mean na 2.8375, ang ikalawa ay 3.0375, ang pangatlong 3.1875, ang pang-apat 2.9625, at ang panlima 2.825. Mula sa mga pahayag, ang una hanggang panglima ay may diskripsyong walang katiyakan kung saan masasabi na ang mga mag-aaral na nasa unang taon na nagpapakadalubhasa sa Filipino ay walang natatanggap na tulong-gabay galing sa kaibigan.

\section{Talahanayan 12}

Suliranin sa pagsagot ng modyul: Guro

\begin{tabular}{|c|c|c|}
\hline Pahayag & Weighted Mean & Diskripsyon \\
\hline $\begin{array}{l}\text { Naglalaan ng oras sa pagtuturo-pagkatuto ng mga } \\
\text { estudyante. }\end{array}$ & 4.1625 & Sumasang-ayon \\
\hline Ibinibigay/ipinapadala ang modyul sa itinakdang oras/araw. & 4.1 & Sumasang-ayon \\
\hline $\begin{array}{l}\text { Nagbibigay ng update/feedback sa kahinaan/kalakasan sa } \\
\text { performansng mga estudyante. }\end{array}$ & 3.75 & Sumasang-ayon \\
\hline Nagbibigay agad ng panuto sa mga gawaing sasagutan. & 4.2125 & Lubos na sumasang-ayon \\
\hline $\begin{array}{l}\text { Mabilis at madali itong sumagot/magbigay-tugon sa mga } \\
\text { paglilinaw. }\end{array}$ & 3.825 & Sumasang-ayon \\
\hline May mga diskusyong ipanapadala sa bawat aralin/paksa. & 3.9125 & Sumasang-ayon \\
\hline $\begin{array}{l}\text { Gumagamit ng iba't ibang estratehiya sa mas mabilisang } \\
\text { pagtuturo at pag-unawa sa mga konsepto. }\end{array}$ & 4.0825 & Sumasang-ayon \\
\hline
\end{tabular}

Ang talahanayan ay resulta ng mga nakalap na datos. Batay sa ipinapakita ng talahanayan, ang unang pahayag ay mayroong weighted mean na 4.1625 na may diskripsyong sumasang-ayon. Sa ikalawa ay mayroong weighted mean na 4.1 na may diskripsyong sumasang-ayon. Samantala, ang pangatlong pahayag ay mayroong weighted mean na 3.75 kung saan ang katumbas na diskripsyon ay sumasang-ayon. Ang ika-apat na pahayag naman ay may weighted mean na 4.2125 na may diskripsyong lubos na sumasang-ayon, ang ikalima ay mayroong weighted mean na 3.825 na may diskripsyong sumasang-ayon, ang ika-anim ay mayroong 3.9125 na weighted mean na may diskripsyong sumasang-ayon at ang pampitong pahayag ay mayroong 4.0825 weighted mean na may diskripsyon na sumasang-ayon. Ibig sabihin, ang mga mag-aaral na nasa unang taon na nagpapakadalubhasa sa Filipino ay nakatatanggap ng sapat na tulong-gabay galing sa kanilang mga guro.

\section{Talahanayan 13}

Suliranin sa pagsagot ng modyul: Kaklase

\begin{tabular}{lcc}
\hline \multicolumn{1}{c}{ Pahayag } & Weighted Mean & Diskripsyon \\
\hline $\begin{array}{l}\text { Tumutugon ito sa mga katanungan o paglilinaw sa isang } \\
\text { paksa/gawain. }\end{array}$ & 3.9875 & Sumasang-ayon \\
$\begin{array}{l}\text { Naipapaliwanag ang mga konseptong hindi ko lubos } \\
\text { maunawaan. }\end{array}$ & 3.8875 & Sumasang-ayon \\
$\begin{array}{l}\text { Mabilis at madali itong sumagot/magbigay-tugon sa mga } \\
\text { paglilinaw. }\end{array}$ & 3.6875 & Sumasang-ayon \\
$\begin{array}{l}\text { Nagpapahiram ng aklat at kagamitan tulad ng cellphone at } \\
\text { laptop. }\end{array}$ & 2.3125 & Walang katiyakan \\
$\begin{array}{l}\text { Nagbibigay ng update sa mga gawaing sasagutan at } \\
\text { proyektong gagawin. } \\
\begin{array}{l}\text { Ibinabahagi ang kanyang kaalaman/natutunan sa isang } \\
\text { konsepto/paksa. }\end{array}\end{array}$ & 3.95 & Sumasang-ayon \\
\hline
\end{tabular}


Edukasyon sa Bagong Kadawyan: Hamon sa pagsagot ng mga mag-aral na nagpapakadalubhasa sa Filipino

Ang talahanayan ay resulta ng mga nakalap na datos. Batay sa ipinapakita ng talahanayan, ang unang pahayag ay mayroong weighted mean na 3.9875 na may sumasang-ayon. Sa ikalawang pahayag naman ay mayroong weighted mean na 3.8875 sumasang-ayon. Samantala, ang pangatlong pahayag ay mayroong weighted mean na 3.6875 kung saan ang katumbas na diskripsyon ay sumasang-ayon. Ang pang-apat na pahayag naman ay may weighted mean na 2.3125 na may diskripsyong walang katiyakan, at ang ikalimang pahayag ay mayroong weighted mean na 3.95 na may diskripsyong sumasang-ayon. Sa panghuli, ay mayroong weighted mean na 3.6625 na may diskripsyong sumasang-ayon. Ibig sabihin ang mga mag-aaral na nagpapakadalubhasa sa Filipino, unang taon, ay nakatatanggap ng tulong-gabay galing sa kanilang kaibigan. Subalit, ang mga mag-aaral ay may isang suliraning kinakaharap sapagkat hindi sila nakahihiram o pinapahiram ng kagamitan na mayroon ang kanilang kaibigan.

\section{Talahanayan 14}

Suliranin sa pagsagot ng modyul: Modyul

\begin{tabular}{lcc}
\hline \multicolumn{1}{c}{ Baryabol } & Weighted Mean & Diskripsyon \\
\hline Disenyo & 4.23 & Lubos na sumasang-ayon \\
Paksa at nilalaman & 4.105 & Sumasang-ayon \\
Gawain at Kahilingan & 3.9660 & Sumasang-ayon \\
Oras & 3.9125 & Sumasang-ayon \\
\hline
\end{tabular}

Ang talahanayan ay ang resulta ng mga nakalap na datos. Batay sa ipinapakita ng talahanayan, ang disenyo ay mayroong weighted mean na 4.23 na may diskripsyong lubos na sumasang-ayon, sa paksa at nilalaman ay may weighted mean na 4.105 na may diskripsyong sumasang-ayon, sa gawain at kahilingan ay mayroong weighted mean na 3.9660 na may diskripsyong sumasang-ayon, at pagdating sa oras ay mayroong weighted mean na 3.9125 na may diskripsyong sumasang-ayon. Ibig sabihin ang mga mag-aaral na nasa unang taon na nagpapakadalubhasa sa Filipino, hinggil sa modyul ay hindi sila nakararanas ng anumang problema o suliranin.

\section{Talahanayan 15}

Suliranin sa pagsagot ng modyul: Pisikal na kalagayan

\begin{tabular}{lcc}
\hline \multicolumn{1}{c}{ Baryabol } & Weighted Mean & Diskripsyon \\
\hline Kagamitan/gadgets & 2.7625 & Walang Katiyakan \\
Internet connection & 2.565 & Hindi sumasang-ayon \\
Kapaligairan/environment & 3.0375 & Walang Katiyakan \\
\hline
\end{tabular}

Ang talahanayan ay resulta ng mga nakalap na datos. Batay sa ipinapakita ng talahanayan, ang kagamitan/gadgets ay may weighted mean na 2.7625 na may diskripsyong walang katiyakan. Sa internet connection naman ay mayroong weighted mean na 2.565 na may diskripsyong hindi sumasang-ayon, at kasunod ang kapaligiran/environment na ay mayroong 3.0375 na may diskripsyong walang katiyakan. Batay sa talahanayan, ang mga mag-aaral na nasa unang taon na nagpapakadalubhasa sa Filipino ay may suliraning kinakaharap pagdating sa pisikal na kalagayan.

\section{Talahanayan 16}

Suliranin sa pagsagot ng modyul: Tulong-gabay

\begin{tabular}{lccc}
\hline & Baryabol & Weighted Mean & Diskripsyon \\
\hline Magulang & 2.735 & Walang katiyakan \\
Kapatid & 2.545 & Hindi sumasang-ayon \\
Kaibigan & 2.97 & Walang katiyakan \\
Guro & 4.0071 & Sumasang-ayon \\
Kaklase & 3.5812 & Sumasang-ayon \\
\hline
\end{tabular}

Batay sa ipinapakita ng talahanayan, ang magulang ay may weighted mean na 2.735 na may diskripsyong walang katiyakan, ang kapatid ay mayroong weighted mean na 2.545 na may diskripsyong hindi sumasang-ayon, at pagdating sa kaibigan ay mayroong weighted mean na 2.97 na may diskripsyong walang katiyakan. Gayunpaman, pagdating sa guro ay mayroong weighted mean na 4.0071 na may diskripsyong sumasang-ayon, at 
Urgil, N., Aninao, J. L., Santo, H., Silvano, L., \& Uñalan, L. A.

sa ikalima mayroong weighted mean na 3.5812 na may diskripsyong sumasang-ayon. Ibig sabihin ang mga mag-aaral na nasa unang taon ay nahaharap sa mga sumusunod na suliranin; tulong-gabay galing sa magulang, kapatid, at kaibigan.

3.2 Ang mga interbensyon sa mga suliranin na kinakaharap ng mga mag-aaral sa pagsagot ng modul:

\section{Talahanayan 17}

Interbensyon sa mga Suliranin sa Pagsagot ng Modyul

\begin{tabular}{lcc}
\hline \multicolumn{1}{c}{ Pahayag } & Frequency & Percentage \\
\hline Nagbibigay agad ng pidbak sa pamamagitan ng text, call, at social media. & 43 & 53.75 \\
Nagbibigay-panuto sa mga gawaing sasagutan. & 22 & 27.5 \\
Nagbibigay ng sapat na oras/araw sa pagbuo ng mga gawain. & 66 & 82.5 \\
Nagbibigay agad ng update sa performance ng estudyante. & 40 & 50 \\
Nilalapatan ng 2-3 halimbawa ang bawat paksa. & 51 & 63.75 \\
Binabawasan ang mga gawaing nakapaloob sa modyul. & 40 & 50 \\
Iniiwasan ang paggamit ng kulay na masakit sa mata, at ang sukat ng mga & 69 & 86.25 \\
salita ay wasto at malaki. & & 36.25 \\
Iniiwasan ang pangkatang gawain. & 29 & 78.75 \\
Blended Learning: synchronous at asynchronous na pagtuturo. & 63 & 41.25 \\
Home visitation once a week. & 33 & \\
\hline
\end{tabular}

Ang talahanayan ay resulta ng mga nakalap na datos. Batay sa ipinapakita ng talahanayan, ang unang pahayag ay mayroong 53.75 bahagdan. Kung sisipatin ang talahanayan ang ikalawang pahayag ay siyang may pinakamaliit na bahagdan at ito ay 27.5 lamang. Ganoon paman, ang ikatlong pahayag ang siyang isa sa may pinakamataas na bahagdan at ito ay mayroong 82.5 bahagdan. Ang ikaapat naman ay mayroong 50 bahagdan, ang ikalima ay 63.75 bahagdan, at ang ikaanim ay 50 bahagdan. Samantala, ang ikapitong pahayag ang siyang may pinakamataas na kung saan ay mayroong 86.75 bahagdan. Mula dito ay sumunod ang ikawalo na mayroong 36.25 bahagdan, ang ikasiyam ay mayroong 78.75 bahagdan at ang huling pahayag ay mayroong 41.25 bahagdan. Sa kabuuan, ang pinakamabisang interbensiyon sa mga suliranin sa pagsagot ng modyul na kinakahaharap ng mga mag-aaral na nasa unang taon na nagpapakadalubhasa sa Filipino ay ang hindi paggamit ng kulay na masakit sa mata. Dagdag pa, ang sukat ng mga salita ay malaki at panatilihin ang kawastuhan ng mga salita. Lumabas din na dapat bigyan ng sapat na oras/araw sa pagsagot at pagbuo ng mga gawain, at higit sa lahat dapat ay maisagawa ang pamamaraang blended learning: synchronous at asynchronous na pagtuturo.

\section{Kongklusyon at Rekomendasyon}

\subsection{Kongklusyon}

Sa pagsaalang-alang sa mga datos ng isinagawang pag-aaral, ang sumusunod na kongklusyon ay nabuo:

$>$ Sa bahagi ng modyul, pagdating sa disenyo, paksa at nilalaman, gawain at kahingian, at oras ay hindi nakararanas ang mga mag-aaral ng suliranin sa pagsagot ng modyul.

> Ganoon pa man, ang mga mag-aaral ay nakararanas ng suliranin pagdating sa kagamitan tulad ng aklat at wifi. Kinahaharap din nila ang mga suliraning pangkapaligiran kung saan maingay ang paligid. Dagdag pa, sila ay nahihirapan sa internet connection.

> Sa pagsagot ng modyul, ang pangunahing suliraning nararanasan ng mga mag-aaral na nasa unang taon na nagpapakadalubhasa sa Filipino ay hindi nila lubusang nauunawaan ang mga konsepto sapagka't wala silang katuwang sa pagsagot ng modyul at kaagapay sa pagpapaliwanag sa mga konsepto.

> Sa mga suliranin sa pagsagot ng modyul na kinahaharap ng mga mag-aaral na nasa unang taon na nagpapakadalubhasa sa asignaturang Filipino, ang interbensiyon sa mga suliranin ay ang pagpapanatili

96 Consortia Academia Publishing (A partner of Network of Professional Researchers and Educators) 
Edukasyon sa Bagong Kadawyan: Hamon sa pagsagot ng mga mag-aral na nagpapakadalubhasa sa Filipino

sa paggamit ng kulay na hindi masakit sa mata. Dagdag pa, ang sukat/laki ng mga salita ay marapat na nasa wastong laki o liit at higit sa lahat panatilihin ang kawastuhan ng bawat salita. Sa mga gawain at kahingian ay magbigay ng sapat na oras/araw sa pagsagot at pagbuo ng mga ito, at higit sa lahat dapat ay maisagawa ang pamamaraang blended learning: synchronous at asynchronous na pagtuturo.

\subsection{Rekomendasyon}

Batay sa mga nabuong kongklusyon, inirekomenda ng pananaliksik ang sumusunod:

$>\quad$ Sa mga kawani ng gobyerno, bigyan ng pansin ang mga nasa mababang sektor ng lipunan pati na rin ang mga paaralan sa mga rural na hindi naabutan ng mga tulong kagamitan sa pag-aaral ng mga kabataan. Dagdag pa, sa mga magulang at opisyal ng barangay, kailangan na bigyan ng atensyon ang kanilang mga anak/kabataan pagdating sa kanilang pag-aaral. Maaari din nilang bigyan ng wifi o maglagay at gumawa ng mga kiosk kung saan tahimik at makakapag-aral sila ng maayos.

$>\quad$ Sa mga instructor/propesor, bigyan ng oras ang pagtuturo sa mga paksa. Upang hindi masayang ang oras, gumawa ng rekording o video presentation sa pagpapaliwanag sa mga konsepto nang sa ganoon ay lubos na mauunawaan ang mga konsepto. Pagdating sa gabay ng pamilya, bigyan ng oras at huwag hayaan na sila'y nahihirapan sa pagsagot ng modyul. Maaari rin silang makipag-ugnayan sa mga guro nang sa ganoon ay epektibo nilang magabayan ang kanilang mga anak.

$>\quad$ Sa mga kagawaran pang-edukasyon, nararapat lamang na mangyari ang blended learning: synchronous at asynchronous na pagtuturo nang sa gayon ay higit na maging epektibo ang pagtuturo-pagkatuto ng mga estudyante sa panahong ito na may pandemya.

\section{Sanggunian}

Almutairy, S. M., Davies, T., \& Dimitriadi, Y. (2015). The readiness of applying m-learning among Saudi Arabian Students at Higher Education. International Journal of Interactive Mobile Technologies (IJIM), $9(3), 33$.

Händel, M., Stephan, M., Gläser-Zikuda, M., Kopp, B., Bedenlier, S., \& Ziegler, A. (2020). Digital readiness and its effects on higher education students' socio-emotional perceptions in the context of the COVID-19 pandemic. Journal of Research on Technology in Education, 1-13.

Hanson, D., Maushak, N. J., Schlosser, C. A., Anderson, M. L., Sorenson, C., \& Simonson, M. (1997). Distance education: Review of the literature (2nd ed.). Washington DC: Association for Educational Communications and Technology.

Kedraka, K., \& Kaltsidis, C. (2020). Effects of the Covid-19 Pandemic on university pedagogy: students' experiences and considerations. European Journal of Education Studies, 7(8).

Krejcie, R. V., \& Morgan, D. W. (1970). Determining sample size for research activities. Educational and Psychological Measurement, 30(3), 607-610.

Musangafi, M. C. C. (2013). Coping mechanisms used by students in open and distance learning (ODL) in response to stressful events. A case study of the Zimbabwe open university (ZOU). IOSR Journal of Agriculture and Veterinary Science, 4(5), 57-63.

Nardo, M. (2017). Modular instruction enhances learner autonomy. American Journal of Educational Research, $5,1024-1034$.

Olamo, T. G., Mengistu, Y. B., \& Dory, Y. A. (2019). Challenges hindering the effective implementation of the harmonized modular curriculum: The case of three public universities in Ethiopia. Creative Education, 10(07), 1365-1382.

Pe Dangle, Y. R. (2020). The implementation of modular distance learning in the Philippine secondary public schools. Proceedings of The 3rd International Conference on Advanced Research in Teaching and Education. 
Urgil, N., Aninao, J. L., Santo, H., Silvano, L., \& Uñalan, L. A.

Sarrab, M. (2015). M-learning in education: Omani undergraduate students' perspective. Procedia - Social and Behavioral Sciences, 176, 834-839.

Vonderwell, S. (2003). An examination of asynchronous communication experiences and perspectives of students in an online course: a case study. The Internet and Higher Education, 6(1), 77-90. 